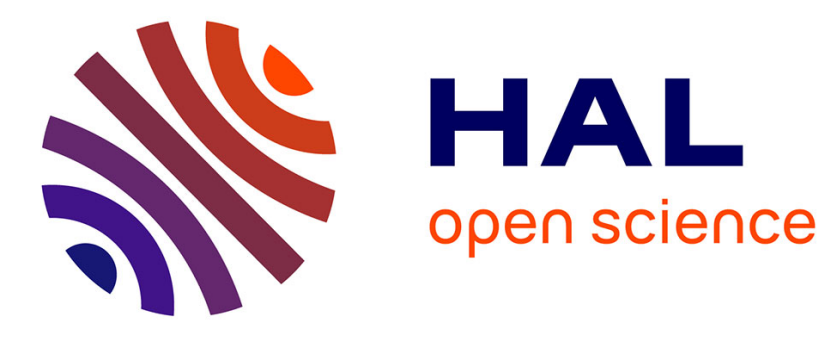

\title{
Molecular organization in hydroperoxidized POPC bilayers
}

Helena Junqueira, André Pierre Schroder, Fabrice Thalmann, Andrey Klymchenko, Yves Mély, Mauricio S Baptista, Carlos M Marques

\section{- To cite this version:}

Helena Junqueira, André Pierre Schroder, Fabrice Thalmann, Andrey Klymchenko, Yves Mély, et al.. Molecular organization in hydroperoxidized POPC bilayers. Biochimica et Biophysica Acta:Biomembranes, 2021, 1863 (10), pp.183659. 10.1016/j.bbamem.2021.183659 . hal-03379156

\section{HAL Id: hal-03379156 \\ https://hal.science/hal-03379156}

Submitted on 14 Oct 2021

HAL is a multi-disciplinary open access archive for the deposit and dissemination of scientific research documents, whether they are published or not. The documents may come from teaching and research institutions in France or abroad, or from public or private research centers.
L'archive ouverte pluridisciplinaire HAL, est destinée au dépôt et à la diffusion de documents scientifiques de niveau recherche, publiés ou non, émanant des établissements d'enseignement et de recherche français ou étrangers, des laboratoires publics ou privés. 


\title{
Molecular organization in hydroperoxidized POPC bilayers
}

\author{
Helena Junqueira, a,: \\ André P. Schroder, ${ }^{\text {, }}{ }^{*}$ Fabrice Thalmann, ${ }^{b}$ \\ Andrey Klymchenko, ${ }^{\mathrm{c}}$ Yves Mély, ${ }^{\mathrm{c}}$ \\ Mauricio S. Baptista, ${ }^{\mathrm{d}}$ \\ and Carlos M. Marques. ${ }^{b}$
}

a. Departamento de Física Aplicada, Instituto de Física, Universidade de São Paulo, CP 66318, 05314970, São Paulo, Brazil.

b. Université de Strasbourg, CNRS, Institut Charles Sadron UPR22, F-67000 Strasbourg, France.

c. Université de Strasbourg, Laboratoire de Bioimagerie et Pathologies, UMR 7021 CNRS, Faculté de pharmacie, 74 route du Rhin, CS 60024, 67401 Illkirch Cedex, France.

d. Departamento de Bioquímica, Instituto de Química, Universidade de São Paulo, 05508-000, São Paulo, Brazil.

† present address : Departamento de Bioquímica, Instituto de Química, Universidade de São Paulo, 05508-000, São Paulo, Brazil

* corresponding author : schroder@unistra.fr

keywords: phospholipid hydroperoxidation, bilayer structure, hydroperoxide localization, hydroxyflavone probes, MARTINI simulations. 


\begin{abstract}
Lipid hydroperoxides are the primary reaction products of lipid oxidation, a natural outcome of life under oxygen. While playing a major role in cell metabolism, the microscopic origins of the effects of lipid hydroperoxidation on biomembranes remain elusive. Here we probe the polar structure of partially to fully hydroperoxidized bilayers of 1-palmitoyl-2-oleoyl-sn-glycero-3-phosphocholine (POPC) by a combination of environment-sensitive fluorescent probes and coarse-grained Martini numerical simulations. We find that the inserted organic hydroperoxide group -OOH migrates preferentially to the surface for bilayers with small fractions of hydroperoxidized lipids, but populates also significantly the bilayer interior for larger fractions. Our findings suggest that by modifying the intimate polarity of biomembranes, lipid peroxidation will have a significant impact on the activity of transmembrane proteins and on the bio-medical efficiency of membrane active molecules such as cell-penetrating and antimicrobial peptides.
\end{abstract}

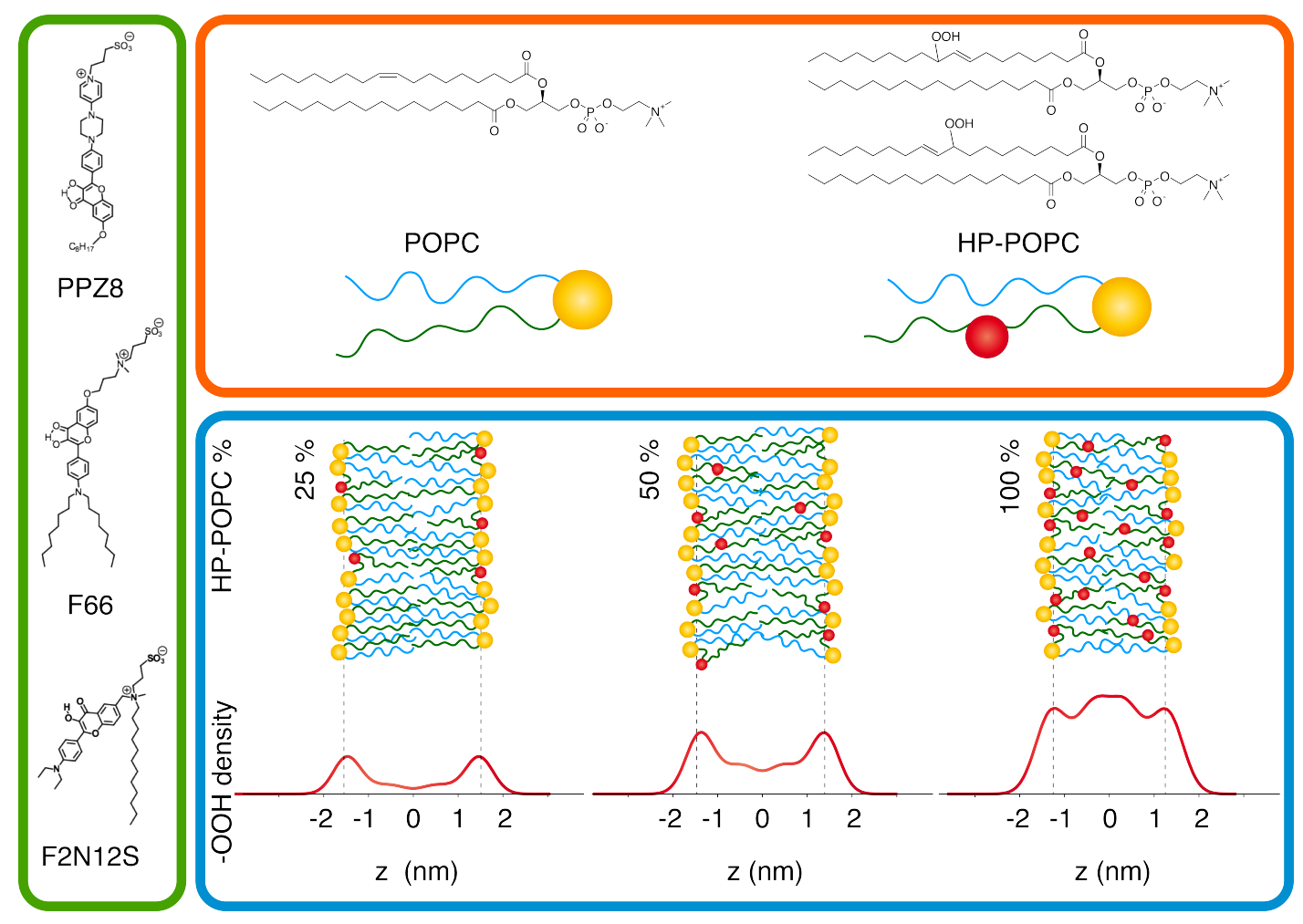




\section{Introduction}

Lipid oxidation occurs during mitochondrial respiration and inflammation, and in several other cellular processes, ${ }^{[1-3]}$ it is also the unavoidable consequence of metabolic activity under light. ${ }^{[4-6]}$ Although a controlled amount of oxidized lipids is required for proper cell signaling, cell maturation and differentiation, and for cell apoptosis, the products of lipid oxidation, if uncontrolled, can have a deleterious effect on the functioning of the cell. Indeed, lipid oxidation products are known to be involved in a variety of neurodegenerative diseases including Parkinson's and Alzheimer's, hypertension and cancer. ${ }^{[7,8]}$

Hydroperoxides are the primary products of lipid peroxidation, which then in vivo serve as substrates for enzymes such as peroxidases and phospholipases, undergoing several secondary reactions. ${ }^{[9]}$ The last decade has seen an increased interest in the effect of lipid hydroperoxidation on the properties of phospholipid membranes. At the molecular level, numerical simulations predicted that insertion of the - $\mathrm{OOH}$ groups changes the statistical distribution of phospholipid conformations, leading noticeably to an increase of the area per lipid.[10,11] Experimentally, oxidation under conditions where hydroperoxides are formed showed consistently an increase in the area per lipid.[12-20] Using for instance a specific Chlorin-derived amphiphilic probe, the in-situ interaction of singlet oxygen ${ }^{1} \mathrm{O}_{2}$ with model membranes of the unsaturated lipids POPC or DOPC (1,2-dioleoyl-sn-glycero-3-phosphocholine) has been studied.[19] Under these conditions the membrane integrity was preserved allowing to measure the area increase associated with the hydroperoxidation of DOPC and POPC bilayers, as well as the reduction of the stretching modulus of partially or totally hydroperoxidized membranes. ${ }^{[19]}$ These results point to possible dramatic changes of the inner structure of the membrane, and in particular to strong changes in polarity, which would have a significant impact on the insertion of membrane proteins or on the interactions with membrane active peptides.

The actual polarity at a given membrane depth, which reflects the local dielectric constant across the direction normal to the bilayer, can be measured using solvatochromic membrane probes. [21,22] Particularly interesting in this respect are membrane probes based on 3-hydroxyflavone (3HF).

[23] Due to excited state intramolecular proton transfer (ESIPT), they exhibit dual emission highly sensitive to the micro-environment. ${ }^{[23-25]}$ Moreover, these dyes can be precisely located at different depths in lipid membranes ${ }^{[26]}$ and they can simultaneously report about polarity as well as hydration (distribution of water across the direction normal to the bilayer).[27] Important examples are the probes used in this study, F2N12S, ${ }^{[28] ~ F 66,[29] ~ a n d ~ P P Z 8[30] ~(F i g . ~ 1) ~ t h a t ~ b e a r ~ z w i t t e r i o n ~ g r o u p s ~ a n d ~}$ 
alkyl chains, which ensures high affinity to lipid and cell membranes and increased sensitivity to their biophysical properties.

In the present work we use F2N12S, F66 and PPZ8 to study membrane polarity at different depths of fully or partially hydroperoxidized POPC bilayers, and coarse-grained Martini numerical simulations to build average $z$-distributions of the -OOH group in the corresponding membranes. Our results show that the hydroperoxide group migrates preferentially to the bilayer interface but also populates significantly the inner membrane region for membrane compositions with more than $50 \%$ of hydroperoxidized lipid content.
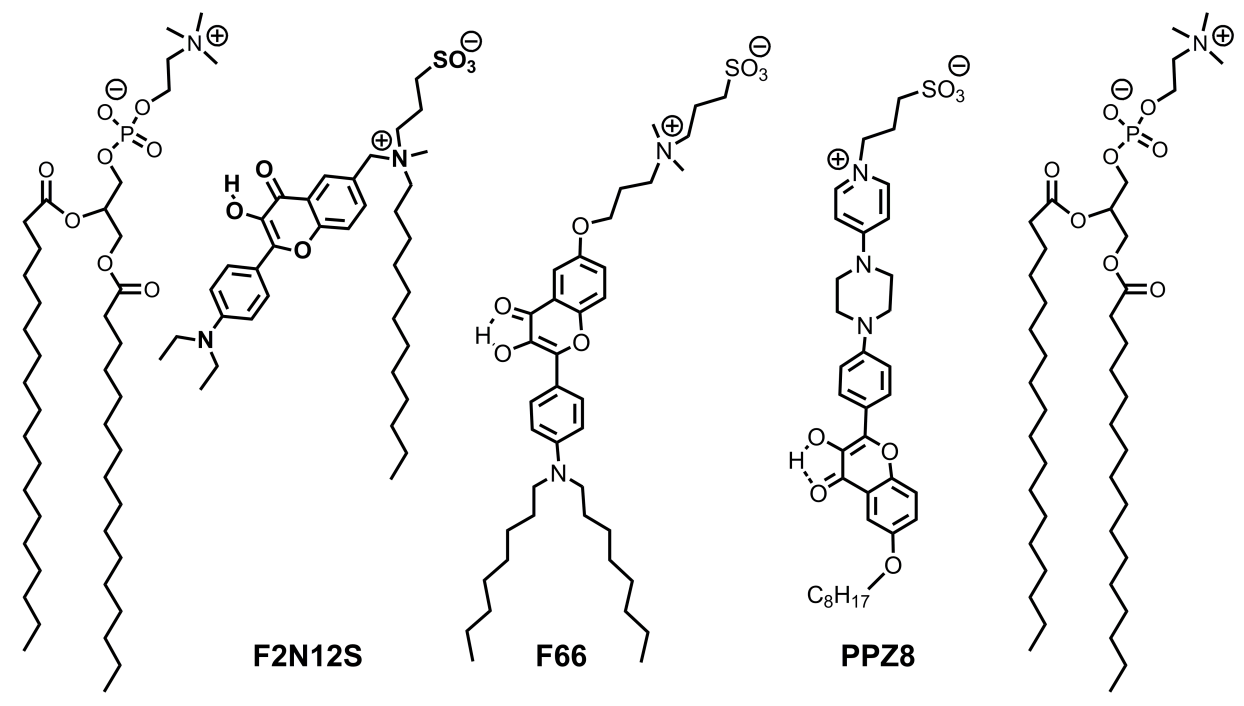

Figure 1: Chemical structures of the three 3-hydroxyflavone (3HF) probes (F2N12S, F66, and PPZ8) and their expected location with respect to lipids in membranes. 


\section{Materials and methods}

\subsection{Materials}

POPC (1-palmitoyl-2-oleoyl-sn-glycero-phosphocholine) was purchased from Avanti Polar Lipids (Alabaster, AL, USA) as a powder. Two POPC in chloroform solutions were prepared; one, of $10 \mathrm{mg} / \mathrm{mL}$ concentration, was used for liposome fabrication. The second, of $16 \mathrm{mg} / \mathrm{mL}$ concentration, was used for HP-POPC fabrication. Both solutions were stored at $-22^{\circ} \mathrm{C}$. All solvents were HPLC grade, and were purchased from Sigma-Aldrich (St. Louis, MO, USA). Methylene blue (MB) was purchased from Synth (Diadema, Sao Paulo, Brazil) in the powder form, and diluted in methanol to a final $0.3 \mathrm{mM}$ concentration. The fluorescent membrane probes F2N12S, F66, and PPZ8 were synthesized as described elsewhere.[28-30] Their chemical structures are presented in Figure 1.

\subsection{Lipid oxidation procedure}

Hydroperoxidized POPC (HP-POPC) was produced as following: first, $80 \mathrm{mg}$ of POPC were dissolved in $5 \mathrm{~mL}$ of chloroform, a solution to which was added $1 \mathrm{~mL}$ of a $0.3 \mathrm{mM} \mathrm{MB}$ in methanol solution, leading to a final mixture of $17.5 \mathrm{mM}$ of POPC and $50 \mu \mathrm{M}$ of $\mathrm{MB}$. This solution was kept in a balloon, under constant stirring and refrigeration using an ice bath, while it was irradiated during $8 \mathrm{~h}$ using a tungsten lamp $(500 \mathrm{~W})$. Then the solvents were rotoevaporated. Finally $1 \mathrm{~mL}$ of methanol was added to the dry residue, and the whole was agitated until its complete dissolution. The solutes of this methanol solution were separated and analyzed with a HPLC-MS (Shimadzu, Kyoto, Japan) system consisting of a shim-pack prep ODS column (150 x $4.6 \mathrm{~mm}, 5 \mathrm{~mm})$, coupled to an Esquire ESI-MS spectrometer. The product that was characterized as POPC hydroperoxide (HP-POPC) had an elution time of $7 \mathrm{~min}$ in a $1 \mathrm{~mL} / \mathrm{min}$ flux and a measured mass of $\mathrm{m} / \mathrm{z}$ 792.7. After elution, the HP-POPC solution was evaporated, re-dissolved in chloroform to a final concentration of $10 \mathrm{mg} / \mathrm{mL}$, and stored at $-22^{\circ} \mathrm{C}$.

\subsection{Liposome fabrication}

Large unilamellar vesicles (LUVs) of POPC, HP-POPC, or of mixtures of both were prepared by the extrusion method. We prepared and studied LUVs made of the following relative POPC/HP-POPC concentrations: 1/0 (pure POPC), 1/1, 3/7, and 0/1 (pure HP-POPC). For that pur- 
pose, we first mixed (when necessary) the appropriate quantities of POPC and HP-POPC chloroform solutions. The mixture was spread in a small flask, and the solvent was evaporated under constant nitrogen flux. Milli-Q water was then added to the dry residue, so to obtain a $200 \mu \mathrm{M}$ lipid suspension. The mixture was gently agitated until the whole residue dispersed in water. This suspension was then extruded 21 times (Avanti Lipids micro extruder) through a $0.1 \mu \mathrm{m}$ filter, obtaining a transparent LUVs suspension. Adding one fluorescent probe (see Fig. 1) to a LUV suspension was achieved by adding the right volume of a $0.3 \mathrm{mM}$ probe-in-DMSO solution, so to reach an overall $0.5 \mu \mathrm{M}$ concentration of the probe (this corresponded for example to the addition of $1.6 \mu \mathrm{L}$ of the probe-in-DMSO solution into $1 \mathrm{~mL}$ of a $200 \mu \mathrm{M}$ LUV suspension), corresponding to a probe/ lipid molar ratio of $0.25 \%$. Addition of a probe was always done just prior to the measurements, as explained below.

\subsection{GUV fabrication}

Giant unilamellar vesicles (GUVs) of pure POPC or of pure HP-POPC were prepared by the electroformation method.[31] Briefly, $5 \mu \mathrm{L}$ of a $1 \mathrm{mg} / \mathrm{mL}$ lipid/chloroform solution were spread onto the conductive side of an ITO covered glass slide (PGO, Germany), forming a lipid stack after the solvent has been evaporated (30 minutes under primary vacuum). Then an electroformation chamber was built by joining this slide, a $1 \mathrm{~mm}$ thick teflon spacer, and a second ITO covered slide, the two conductive sides facing each other. The cell was then filled with a $100 \mathrm{mOsm}$ sucrose/water solution, and an electric alternate field was applied to the cell during 2 hours $(1 \mathrm{~V}, 10 \mathrm{~Hz})$. Finally, the GUVs were extracted from the growing cell and dispersed into a glucose-containing microtube (Eppendorf, Germany) for further use (approximate dilution 1/1). Sucrose and glucose solutions were osmotically matched (100 mOsm), using a cryoscopic osmometer (Osmomat 030, Gonotec, Germany). Thanks to a difference in density, the sucrose-containing GUVs sedimented rapidly in their environing glucose-sucrose solution, facilitating their further use and observation. It is worth to note that, contrary to the case of LUVs (see above), the phospholipid quantity present in a GUV suspension is not known, since part of the initial lipid remains attached to the ITO glass surface.

Just before their observation, a DMSO solution containing a flavone-based probe was added to the GUV suspension assuming that $20 \%$ of the lipids initially present in the growing chamber have been collected, with the aim of reaching a final probe/lipid molar fraction of $0.25 \%$. The GUV suspension was then transferred into an observation chamber, and observed under the spectral imag- 
ing confocal Nikon microscope (see below). Since the real probe/lipid fraction could not be controlled precisely, no quantitative measurements of fluorescence intensity were done on GUVs. Spectral imaging was therefore performed with the purpose of checking the effectiveness of probe insertion in the bilayers and of qualitatively comparing with fluorescence analysis on LUVs.

\subsection{Spectral imaging confocal microscopy}

We used a TE2000 inverted microscope, equipped with a 100x oil-immersion objective, and a C1 confocal scanning head connected to a 32-channel Spectral Imaging C1-si System (Nikon, Japan). The device enabled us to create in a single scan of the laser a 32 channels spectral image of the sample. We acquired sets of 32 images of flavone-based probe-containing GUVs in the range 435-745 $\mathrm{nm}$ (i.e. with $10 \mathrm{~nm}$ broad channels). Excitation wavelength was set at $408 \mathrm{~nm}$. Figure 2 shows spectra of pure POPC GUVs and pure HP-POPC GUVs labeled with the F66 probe, each spectrum being the average over ten GUVs. ImageJ software (https://imagej.nih.gov/ij/) was first used to integrate the light intensity along each GUV perimeter and then used to extract the GUV spectrum. Inset in Fig. 2 shows typical F66-containing GUVs (here made of HP-POPC). Please note that we never observed any sign of in situ oxidation during fluorescence imaging, something that can eventually take place with some fluorescent probes, as for example rhodamine- and NBD-based probes. $[32,33]$ 


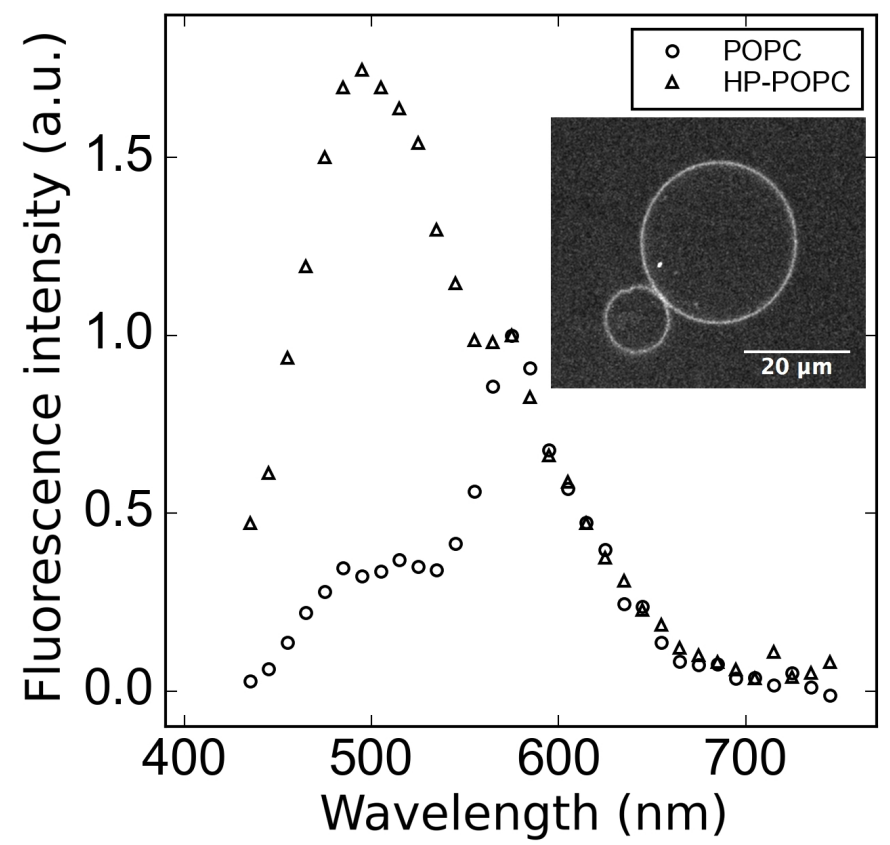

Figure 2: Normalized fluorescence spectra of giant unilamellar vesicles incorporating the F66 probe. Averaged spectra of ten pure, 100\% POPC, and of ten pure, 100\% HP-POPC GUVs. The curves are normalized to their $\mathrm{T}^{*}$ pick value. Inset: a typical fluorescence image, here obtained as the sum of the 32 images measured by the 32-channels of the C1-si spectral microscope (HP-POPC GUVs are shown here).

\subsection{Fluorescence spectra of LUVs, acquisition and analysis}

Adding one of our 3-hydroxyflavone probes in a LUV suspension (see above) was done directly in a dedicated, 1-cm path length quartz cuvette (HELLMA, Müllheim, Germany). After probe addition, the cuvette was gently agitated. Then, fluorescence spectra were immediately recorded on an Agilent Cary Eclipse Fluorescence Spectrophotometer (USA), with an excitation wavelength of $408 \mathrm{~nm}$. All experiments were performed at room temperature. A typical fluorescence spectrum is given in Figure 3. Deconvolution of the spectra into three bands $\left(\mathrm{N}^{*}, \mathrm{H}-\mathrm{N}^{*}\right.$ and $\left.\mathrm{T}^{*}\right)$ was done with a homemade program developed in python (https://www.python.org/). The program is based on an iterative nonlinear least-squares method, that is itself based on the Fletcher-Powell algorithm, following a procedure already reported.[34] The individual emission bands were approximated by a $\log$ normal function that accounts for several parameters (maximal amplitude, $I_{\max }$, spectral maximum 
position, $\lambda_{\max }$, and positions of half-maximum amplitudes, $\lambda_{1}$ and $\lambda_{2}$, for the blue and red parts of the band, respectively). The program operates with the shape parameters of a log-normal function, such as the full width at the half-maximum, FWHM $\left(\lambda_{1}-\lambda_{2}\right)$, and band asymmetry, $\mathrm{P}=\left(\lambda_{1}-\lambda_{\max }\right) /$ $\left(\lambda_{\max }-\lambda_{2}\right)$, and allows approximating the asymmetric bands in absorption and fluorescence spectra. To obtain more stable results for the deconvolution, several parameters were fixed based on previous data in organic solvents and LUVs. ${ }^{[35]}$ Thus, the FWHM of the two short-wavelength bands $\left(\mathrm{N}^{*}\right.$ and $\mathrm{H}-\mathrm{N}^{*}$ ) were fixed at $3000 \mathrm{~cm}^{-1}$. For the $\mathrm{H}-\mathrm{N}^{*}$ band, the asymmetry was fixed at 0.9 , whereas the band position was fixed at $18,400 \mathrm{~cm}^{-1}$. The other parameters, i.e., asymmetry of $\mathrm{N}^{*}$ and $\mathrm{T}^{*}$ bands, width of the $T^{*}$ band and relative intensities of the bands, were allowed to vary. An example of deconvolution is presented in Figure 3. The fluorescence intensities of the separated $\mathrm{N}^{*}, \mathrm{H}_{-} \mathrm{N}^{*}$ and $\mathrm{T}^{*}$ bands $\left(\mathrm{I}_{\mathrm{N}^{*}}, \mathrm{I}_{\mathrm{H}-\mathrm{N}^{*}}\right.$, and $\left.\mathrm{I}_{\mathrm{T}^{*}}\right)$ were used for calculation of $\left.\mathbf{i}\right)$ the hydration parameter HYD and ii) the membrane polarity POL. HYD was expressed as the ratio of the emission intensity of the hydrated $\left(\mathrm{H}-\mathrm{N}^{*}\right)$ form to the summed intensities of the non-hydrated $\left(\mathrm{N}^{*}\right.$ and $\left.\mathrm{T}^{*}\right)$ forms, taking into account that the FWHM for the $\mathrm{T}^{*}$ band is 2.5 -fold narrower than for the $\mathrm{N}^{*}$ and $\mathrm{H}-\mathrm{N}^{*}$ bands. Thus one gets $\mathrm{HYD}=\mathrm{I}_{\mathrm{H}-\mathrm{N}^{*}} /\left(\mathrm{I}_{\mathrm{N}^{*}}+0.4 \times \mathrm{I}_{\mathrm{T}^{*}}\right)$, and $\mathrm{POL}=\mathrm{I}_{\mathrm{N}^{*}} / \mathrm{I}_{\mathrm{T}^{*}}$, as derived elsewhere.[27] As a matter of fact, HYD parameter is related to free water molecules available for H-bonding with the probe, the $\mathrm{H}-\mathrm{N}^{*}$ form of the probe being located shallow in the glycerol ester region of the bilayer, reflecting the membrane hydration.[27] On the contrary, $\mathrm{N}^{*}$ and $\mathrm{T}^{*}$ correspond to a deeply inserted form of the probe, the POL parameter being related to dipoles present in the inner membrane environment, including both free and bound water, and carbonyl or hydroperoxide (-OOH) groups.[27,35] 


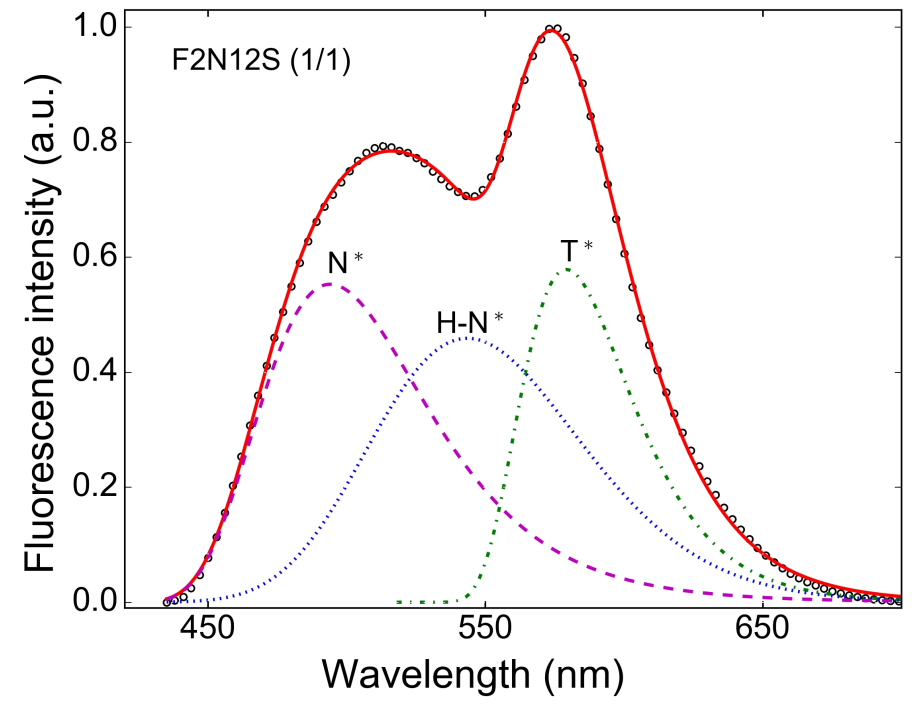

Figure 3: Black circles : normalized emission spectrum of F2N12S-incorporating LUVs made of 1/1 POPC/HP-POPC. Continuous red line : best fit. Dashed, dotted, dot-dashed lines correspond to the decomposition of the best fit into the three (log-normal) $\mathrm{N}^{*}, \mathrm{H}-\mathrm{N}^{*}$ and $\mathrm{T}^{*}$ bands respectively.

\subsection{Fluorescence lifetime}

A Time-resolved Confocal Fluorescence Microscope (Olympus IX71) with Unique Single Molecule Sensitivity (Picoquant, Microtime 200, PicoQuant, Berlin, Germany) equipped with a FLIM system (pulsed laser diode LDH 405, operating at $405 \mathrm{~nm}$, with a pulse width $<64 \mathrm{ps,} \mathrm{fre-}$ quency $80 \mathrm{MHz}$ ) and with a 60x water immersion objective was used (UPlanSApo, water immersion, $\mathrm{WD}=0.28 \mathrm{~mm}$ ). Fluorescence was directed through a $50 \mu \mathrm{m}$ pinhole and detected with a single-photon avalanche diode (SPAD, SPCM-AQR-13, PerkinElmer, Waltham, USA), after passing through a 580/70-BP filter (corresponding to the wavelength range 545..615 nm). Time-resolved records were made in single-photon counting mode using the TimeHarp 260 PICO platform (PicoQuant, Berlin, Germany), resolution $<25$ ps. Experiments were done on LUV suspensions prepared as described above. Briefly, a $50 \mu \mathrm{L}$ droplet of a 3HF-labelled LUV suspension was dropped in an observation chamber made of a coverslip, a $1 \mathrm{~mm}$ thick silicon spacer, and a top glass slide. Image acquisition was done using the SymPhoTime64 software version 2.1.3813. Thanks to the small (100 $\mathrm{nm}$ ) size of LUVs, the FLIM images showed an equally distributed fluorescence over the whole observation field. The lifetimes were thus calculated by averaging over the whole observation field. 


\subsection{Numerical simulations}

We performed molecular dynamics simulations of a bilayer comprising POPC and hydroperoxidized HP-POPC. We use the Martini Coarse-Grained model, for which a model of POPC is available ${ }^{[36]}$ and a recently introduced coarse-grained (CG) model of hydroperoxide described in reference. ${ }^{[11]}$ Both coarse-grained molecules are shown in Fig. 4, while details regarding the coarsegraining procedure can be found in.[36] We briefly list here the chemical nature of the beads appearing in Fig. 4. For POPC: bead 1 choline, 2 phosphate, 3 and 4 glycerol and ester groups, 5-8, 9-10, 12-13 butane like tail components, 11 cis-butene group with $120^{\circ}$ bending angle. For HP-POPC: same groups except but 11 trans-butene, and 11p hydroperoxide $\mathrm{OOH}$. Martini-like coarse graining does not distinguish between $2 n$ and $2 n+2$ carbons in the aliphatic chains. As emphasized in, ${ }^{[11]}$ peroxidation reactions create a mixture of compounds with hydroperoxide groups added either above or below the oleoyl insaturated bonds. Our model aims at representing both species as one.
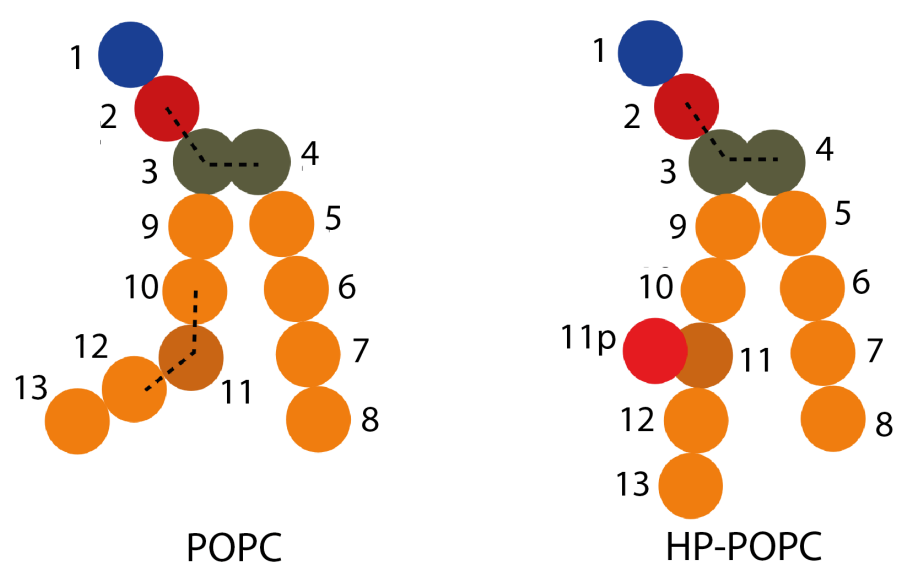

Figure 4: Coarse graining of POPC and hydroperoxidized HP-POPC molecules. The POPC lipid corresponds to the standard Martini model, with a 4 beads tail for the palmitoyl and a 5 beads tail for the oleoyl fatty acids. Upon peroxidation, the POPC bead 11 (cis-butene) is replaced by a hydrophilic-hydrophobic pair of beads (11,11p in the HP-POPC model). The "hydroperoxide" bead $11 \mathrm{p}$ serves to determine the local density of hydroperoxide groups in the membrane. See text for a detailed list of bead chemical correspondence.

Our bilayer comprised a total of 512 lipids, with a symmetric leaflet composition. Our simulated systems were made respectively of 16 HP-POPC and 496 POPC (3.125 \% oxidation), 128 HPPOPC and 384 POPC (25\% oxidation), 256 HP-POPC and 256 POPC (50 \% oxidation), 358 HP- 
POPC and 154 POPC (70 \% oxidation) and 512 HP-POPC (100\% oxidation). Compared with the original publication, [11] we used a larger number of water molecules, i.e. 4272 water beads corresponding to 8.25 water beads (or 33 water molecules) per lipid in this coarse-graining scheme. As a matter of fact it was realized since[11] that hydroperoxidized lipid bilayers require more water to be fully hydrated than usual bilayers.

An integration step of 40 fs was used, along with a Parinello-Rahman semi-isotropic barostat and velocity-rescale thermostat, as provided by the molecular dynamics engine Gromacs, version 4.6.2. Lipid parameter files were martini_v2.1.itp, and martini_v2.0_lipids.itp. A total of $4 \mathrm{x}$ $10^{7}$ steps corresponding to $0.16 \mu$ s of CG simulation time was used to compute the density distribution of the bead 12, representative of the hydroperoxide groups in our model (Fig. 4). Frames were recorded every 1000 steps, out of which a density profile was calculated with the Gromacs tool $g_{-}$density. Each trajectory was split into 4 parts. For each part ( $10^{7}$ steps), the bilayer center was estimated and shifted in order to obtain histograms exactly centered around $z=0$, where $z$ represents the axis perpendicular to the membrane mid-plane. This is because a slight drift of the bilayer along the $z$ direction may occur as a result of spontaneous random crossing events of water beads, facilitated by hydroperoxidation. A total of 50 bins was used for the $z$-distribution in each histogram. Partitioning of the hydroperoxide groups between core and interface was derived from the above histograms, with the core region arbitrarily defined by the geometrical condition $|z|<z_{c}=0.8 \mathrm{~nm}$. The interface corresponds in our model to the region characterized by $z_{c}<|z|<z_{i}=2.5 \mathrm{~nm}$.

The deuterium order parameter, $S_{C D}$, is a measure for the order of the lipid tails in the bilayer.[37] $S_{C D}$ is itself defined from $S_{C D, n}$ the order parameter of an individual C-D bond :

$$
S_{C D, n}=\frac{1}{2}\left(3\left\langle\cos ^{2}\left(\theta_{C D, n}\right)\right\rangle-1\right)
$$

$\theta_{C D, n}$ being the angle between the n-th $\mathrm{C}-\mathrm{D}$ (or $\mathrm{C}-\mathrm{H}$ ) bond and the normal to the bilayer, the $z$-axis. Thus defined, $S_{C D, n}$ ranges between -1/2 (segments lying in the $x y$ plane) and 1/4 (segments parallel to $z$ ). Such definition can be extended to the molecular representation in a CG method. First, consider that, if one excepts terminal methyl (-CH3) groups, the $\mathrm{C}_{\mathrm{n}} \mathrm{H}$ bond orientation associated with any 3 consecutive carbon atoms $\mathrm{C}_{\mathrm{n}-1}, \mathrm{C}_{\mathrm{n}}, \mathrm{C}_{\mathrm{n}+1}$ along a chain can be related to the segment orientation $\mathrm{C}_{\mathrm{n}-1} \mathrm{C}_{\mathrm{n}+1}$. As coarse-grained models do not explicitly feature hydrogen atoms and cluster together many carbon atoms into one bead, we take the bead segments $B_{n-1} B_{n+1}$ as representative of the car- 
bon-carbon segments $(\mathrm{C}-\mathrm{C})$, with $\mathrm{B}_{\mathrm{n}}$ the bead number $\mathrm{n}$ according to Fig. 4 . Denoting $\theta_{C C, n}$ the angle between the segment $\mathrm{B}_{\mathrm{n}-1} \mathrm{~B}_{\mathrm{n}+1}$ and the bilayer normal $z$, an index dependent order parameter can be defined similarly to eq.(1) :

$$
S_{C C, n}=\frac{1}{2}\left(3\left\langle\cos ^{2}\left(\theta_{C C, n}\right)\right\rangle-1\right)
$$

Thus defined, $S_{C C, n}$ ranges between 1 (segments lying in the $x y$ plane) and -1/2 (segments parallel to $z$ ). One thus gets the relation : $S_{C D, n}=-1 / 2 S_{C C, n}$. Similarly to $S_{C D}$, a global order parameter $S_{C C}$ for a given POPC:HP-POPC composition was computed by averaging $S_{C C, n}$ (eq. 2) over the index $n$ and over the chemical species concentrations. For both POPC and HP-POPC molecules, the statistics is performed on beads number 5,6,7,9,10,11,12 (Fig. 4). In practice, the gmx order Gromacs software utility was used to compute $S_{C C}$. For convenience, $-S_{C D}=1 / 2 S_{C C}$ will be given here, so that our values can be directly compared to those of literature. Isotropic distribution corresponds to a vanishing value of the order parameter, while positive decreasing values of $-S_{C D}$ express a reduction of membrane ordering. 


\section{Results and discussion}

\subsection{Fluorescence analysis}

The three probes are non-fluorescent in water and become highly fluorescent on binding to phospholipid membranes.[28] This was in our case confirmed by imaging GUVs by confocal microscopy: GUVs appear as fluorescent capsules in a dark, non-fluorescent environment (inset in Fig. 2). The fluorescence of the three probes bound to lipid membranes is characterized by two emission bands, which, according to previous studies of 3-hydroxyflavones, $[26,27,35]$ can be assigned to $\mathrm{N}^{*}$ and $\mathrm{T}^{*}$ forms for the short and long-wavelength bands, respectively. Figure 2 compares a set of pure POPC and a set of pure HP-POPC GUVs incorporating the F66 probe, each spectrum being an average over ten GUVs. In HP-POPC, the emission spectrum of F66 is dominated by the N* band, while in POPC bilayers, the $\mathrm{T}^{*}$ band is dominant. Similar results were obtained with the two other flavone-based probes. However, although averaged over several GUVs, the confocal microscopy spectra lack precision, due partly to the variation of the probe/lipid fraction in the bilayers. More reproducible results were obtained from spectral analysis of LUVs suspensions by fluorescence spectroscopy. Figure 5 shows the normalized fluorescence spectra of probe-containing LUVs. Relative POPC/HP-POPC molar ratios 1/0, 1/1, 3/7, and 0/1 have been studied. For each probe, increasing HP-POPC concentration in the bilayer results in a significative modification of the fluorescence spectra, with a clear increase of the intensity in the $500 \mathrm{~nm}$ region (Fig. 5). Deeper analysis was obtained from the deconvolution of the spectra into the three $\mathrm{N}^{*}, \mathrm{~T}^{*}$ and $\mathrm{H}-\mathrm{N}^{*}$ bands (typical example in Fig. 3). The overall modification of the spectra with HP-POPC fraction (Fig. 5) appeared to be mostly related to a significative increase of the $\mathrm{N}^{*} / \mathrm{T}^{*}$ ratio, while some increase in the intensity of the H-N* pic was also observed (not shown). Results of deconvolutions expressed in parameters of polarity (POL) and hydration (HYD) are given in Figure 6. Each probe reports a nonlinear (roughly quartic) increase of the local polarity as a function of HP-POPC concentration, as shown in Figure 6A. PPZ8, the probe most deeply inserted in the membrane (Fig. 1), exhibits a polarity value close to zero for low HP-POPC fractions and the highest relative increase in the polarity parameter (Fig. 6A). Contrary to polarity, the hydration parameter remains unchanged (within experimental error) with increase in the HP-POPC fraction (Fig. 6B).

Relative variations of the $\mathrm{N}^{*}$ and the $\mathrm{T}^{*}$ band intensities of $3 \mathrm{HF}$ probes inserted in model membranes have been widely reported for different membrane compositions. ${ }^{[27,35,38]}$ For example, increase of the unsaturated/saturated lipid fraction results in the increase of the $\mathrm{N}^{*} / \mathrm{T}^{*}$ ratio, while increase of the cholesterol content results in the decrease of the $\mathrm{N}^{*} / \mathrm{T}^{*}$ ratio. ${ }^{[27]}$ Also, transition from 
gel to fluid state induces a strong increase of $\mathrm{N}^{*} / \mathrm{T}^{*}$, while increasing the temperature of a fluid bilayer results in the increase of HYD parameter only, POL remaining unchanged.[27] As a matter of fact, hydration and polarity are not always coupled, as seen with the latter example, and as reported in some other particular cases.[27,35] POL parameter, i.e. $\mathrm{N}^{*} / \mathrm{T}^{*}$, reflects the polarity changes in the close environment of the deeply inserted probe, including water presence, that is known to be related to the order parameter of the lipids. HYD parameter, that is calculated as a relative increase of the $\mathrm{H}-\mathrm{N}^{*}$ band with respect to a weighted sum of both $\mathrm{N}^{*}$ and $\mathrm{T}^{*}$ (see MM section) reflects the bilayer hydration in the interface region, $\mathrm{H}-\mathrm{N}^{*}$ corresponding to a shallow conformation of the probe. [27] Here, depending on its position with respect to the membrane depth, a grafted OOH may either create an $\mathrm{H}$-bond with a probe in its shallow form, changing its $\mathrm{H}-\mathrm{N}^{*}$ state, or modify strongly the polar environment of a deeply inserted probe, influencing $\mathrm{N}^{*} / \mathrm{T}^{*}$. The shallow region is highly polar and hydrated, we observe that HYD parameter is not influenced by the $\mathrm{OOH} / \mathrm{H}-\mathrm{N} *$ interaction in that region (Fig 6B). Deeper in the membrane, the presence of $\mathrm{OOH}$ might induce a modification of the local disorder, and thus increase the quantity of water, something predicted to some extent by simulations, see below. Also, $\mathrm{OOH}$ groups may interact directly with the probe, or modify their polar environment, both influencing $\mathrm{N}^{*} / \mathrm{T}^{*}$. The observed POL variation (Fig. 6A) may thus have several origins, $\mathrm{OOH}$ group acting certainly as the driver. Thus, Fig. 6A tells us that only for HPPOPC molar fractions above 50\% does the polarity in the membrane start to increase, suggesting that at lower HP-POPC fractions, the hydroperoxides mainly populate the interface. 


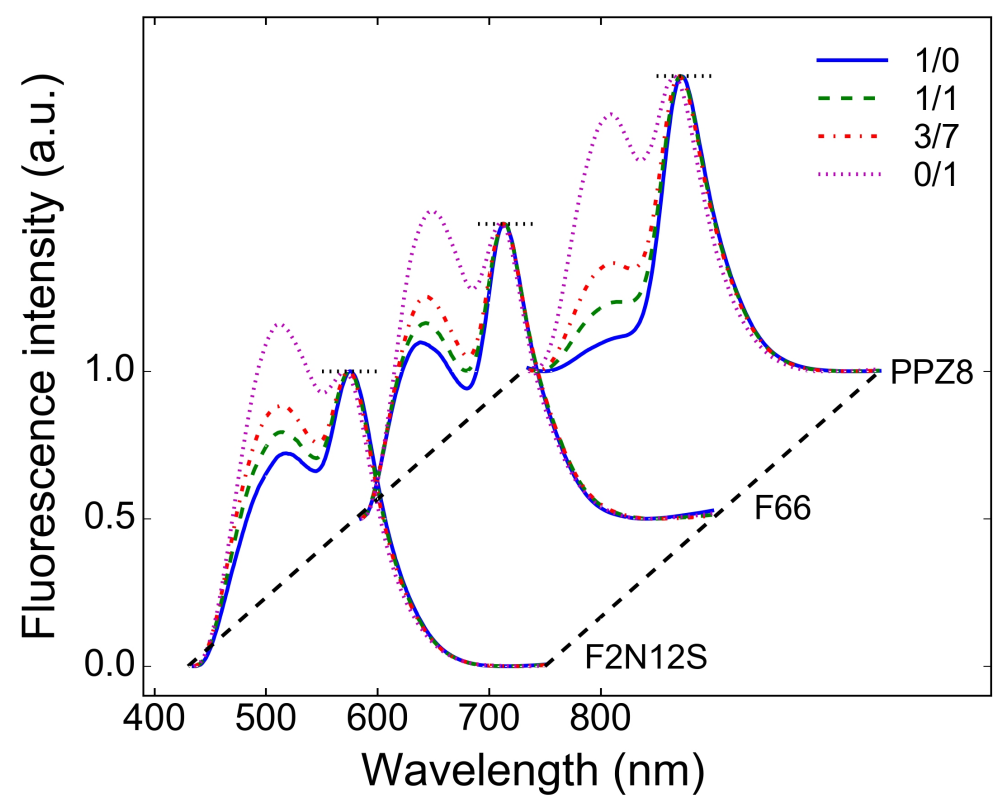

Figure 5: Fluorescence spectra of LUVs incorporating the 3HF probes. Four membrane compositions are studied, expressed as the POPC/HP-POPC relative fraction: $1 / 0$, i.e. a pure POPC membrane (full line), 1/1 (dashed), 3/7 (dot-dashed), and 0/1 (dotted), corresponding to a pure HP-POPC membrane. Signals from the three probes F2N12S, F66, and PPZ8 are normalized to their value for the $\mathrm{T}^{*}$ pick.
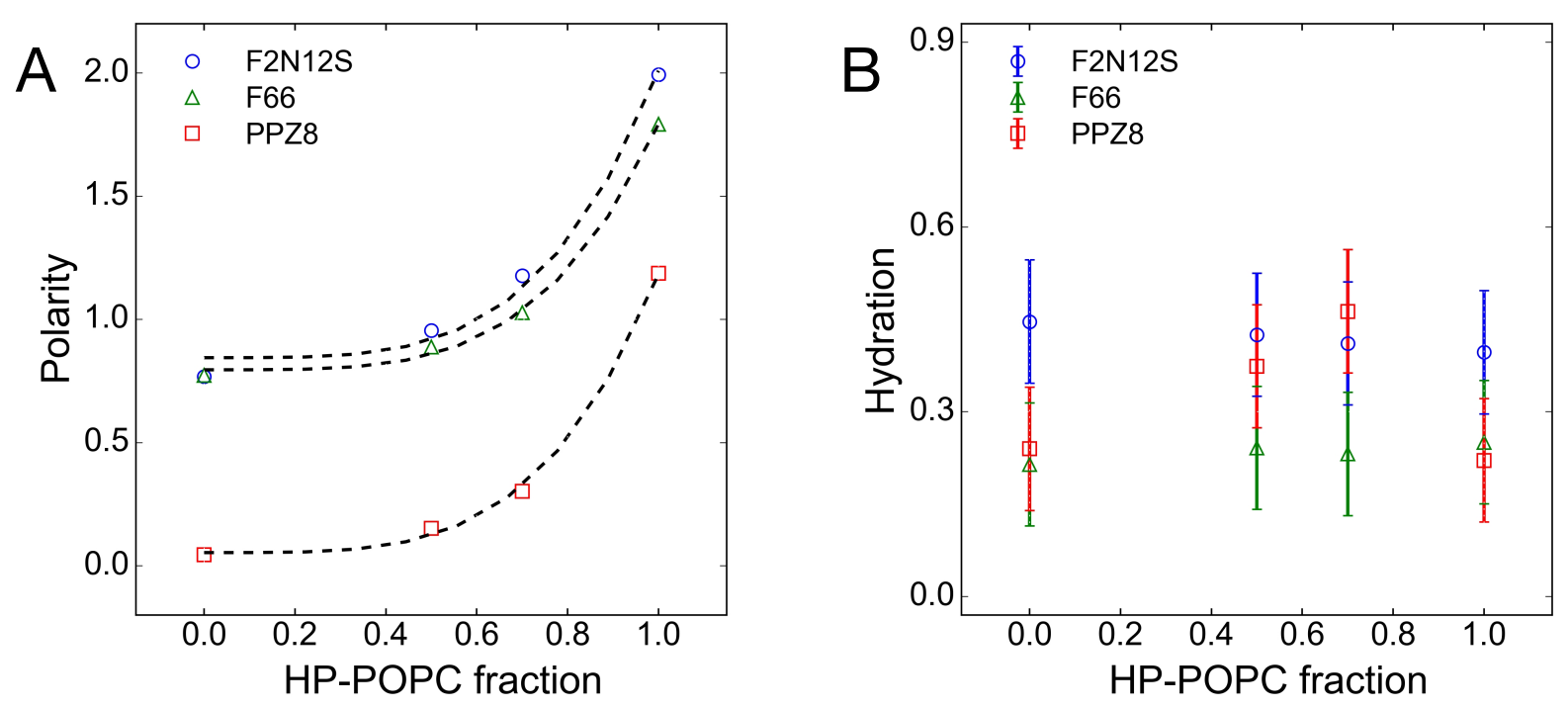

Figure 6: (A) local polarity coefficient (POL) for each of the 3HF probes, as a function of the HPPOPC fraction. Dashed lines correspond to best fits with a quartic polynomial. (B) hydration coefficient (HYD) for each of the 3HF probes, as a function of the HP-POPC fraction. 
Dependance of POL and HYD parameters on local micro-environment for various 3-HF probes have already been observed in other cases. For example, the sensitivity of F2N8 and FN4, the first with a charged anchor group, binding preferentially to the lipid membrane interface, and the second highly hydrophobic, have been compared in three model environments : the DOPC bilayer, Hepatitis B surface antigen (HBsAg) particles, and low density lipoproteins (LDLs) [39]. Fig. 5 for F2N8 (resp. Fig. 6 for FN4) in [39] show variations of a factor of 2 (resp. 3) for the POL parameter, when the probes are inserted in LDL or in HBsAg. This is coherent with the compositions of those particles : LDLs contain a hydrophobic fluid core with mainly triglycerides and fatty acids, while the core of HBsAg is mainly composed of proteins. POL is then clearly related to local polarity, that is expected to be higher around proteins than inside triglycerides. Also, FN4, the most hydrophobic probe, is more sensitive than F2N8 to the core composition of LDLs and HBsAg [39], similarly to what is observed here with PPZ8, when compared to F2N12S and F66.

To gain further information on the HP-POPC-containing membranes, the fluorescence lifetimes of the $\mathrm{T}^{*}$ band of the $3 \mathrm{HF}$ probes were recorded (Figure $7 \mathrm{~B}$ ). In the case of F2N12S, these lifetimes were shown to be exquisitely sensitive to the lipid order phase, with a two-fold increase in the fluorescence lifetime for the liquid order (Lo) phase as compared to the liquid disorder (Ld) phase.[40] This two-fold increase was related to the restriction by the highly packed Lo phase of the relative motions of the two aromatic moieties of the $3 \mathrm{HF}$ fluorophore, which favors the highly emissive flat conformation of the probes.[40] For membranes with a molar fraction of HP-POPC lower than $80 \%$ the three probes exhibit one unique lifetime, equal to $\tau=3.7 \pm 0.1 \mathrm{~ns}$, which is typical of the Ld phase. However, for pure HP-POPC membrane, the three probes exhibit a second, longer lifetime (also detected for the 20/80 composition for F2N12S), typical of Lo phase. ${ }^{[40]}$ As seen in Fig. 7B, the uncertainty on this second lifetime is high, due its low contribution to the total FLIM signal (see Fig. 7A, for a typical example). The minimal changes in the lifetime observed here for $0-80 \%$ of HP-POPC indicate that the probes did not detect strong variation in the lipid order. Thus, the observed ratiometric response of these probes to the presence of HP-POPC (Fig. 6A) should be mainly connected with the increase in the environment polarity.

Figure 7: (A): FLIM signal for pure HP-POPC LUVs, incorporating F66 probe. Excitation and emission wavelength were $405 \mathrm{~nm}$ and [545..615] $\mathrm{nm}$ respectively. Dashed line is the best fit for a 

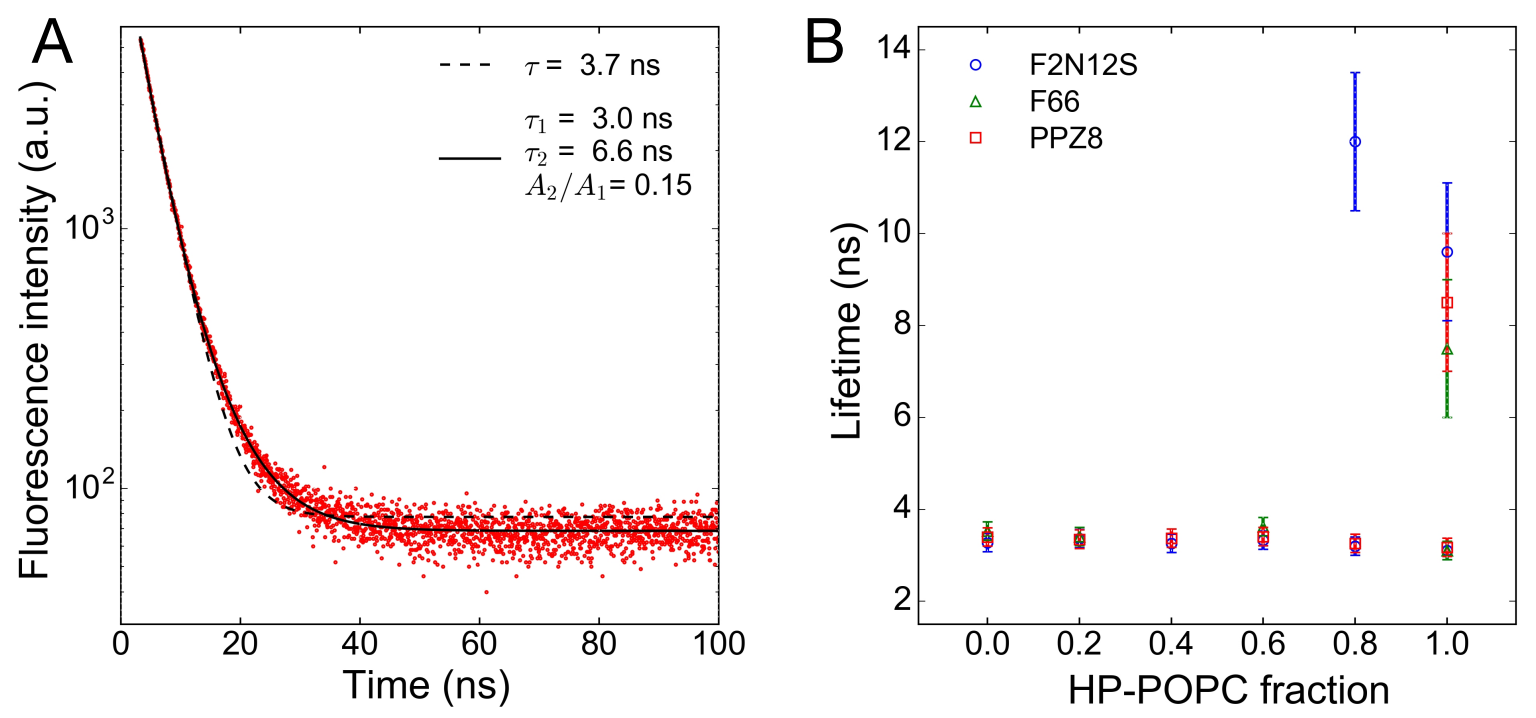

single time decrease, i.e. $\mathrm{A} \exp (-\mathrm{t} / \tau)$, while continuous line is for the best fit with a 2-lifetimes function, i.e. $\mathrm{A}_{1} \exp (-\mathrm{t} / \tau 1)+\mathrm{A}_{2} \exp (-\mathrm{t} / \tau 2)$. (B): lifetimes for pure and mixed membranes of POPC and HP-POPC. Only at the higher HP-POPC fractions does appear a second, longer lifetime in the FLIM signal (as exemplified in A).

\subsection{Numerical simulations}

As discussed above, the increase of the $\mathrm{N}^{*}$ band with HP-POPC fraction (Fig. 5) is certainly associated to an increase of the number of dipoles in the deep membrane. Both water molecules or hydroperoxide groups can induce the corresponding increase of POL parameter shown in Fig. 6A. Numerical simulations aim at clarifying this point. On the one hand, an increased water presence in the deep membrane is associated to an increased disorder of the bilayer. As a matter of fact our simulations show a slight decrease in lipid order with peroxydation (Fig. 8A): $-S_{C D}$ decreases in the range [0.21..0.14] for increasing HP-POPC fraction from $0 \%$ to $100 \%$. Data in Fig. 8A can be compared to those obtained by Van der Paal et al.[37] using All Atom simulations (AA), see Fig. 3d in their article: their $-S_{C D}$ decreases over a comparable range, namely [0.16..0.12]. We give in Supplementary Information the evolution of individual $-S_{C D, n}$ for each of the beads of the carbon chain of both POPC and HP-POPC, with HP-POPC fraction in the bilayer (Fig. S1). While for beads of the saturated (sn-1) chain (beads 5,6,7), $-S_{C D, n}$ decreases slightly over the range [0.3..0.2] with peroxidation for both POPC and HP-POPC, beads of the unsaturated chain (sn-2) of HP-POPC (beads 9-12) surprisingly evolve oppositely, i.e. from a more disordered configuration for a membrane with low HP-POPC fraction, towards a more ordered configuration for a fully peroxidized 


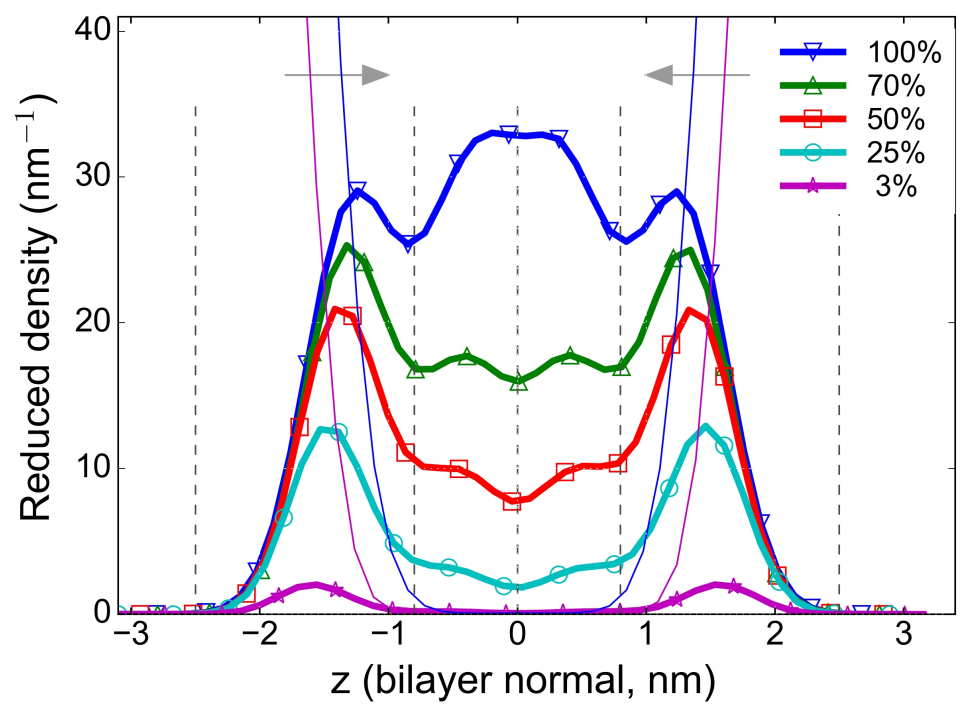

bilayer. Note that for the sn-2 chain of POPC, $-S_{C D, n}$ values are similar to their sn-1 counterpart, except for bead 12 (Fig. S1). On the other hand, hydroperoxyl groups (-OOH) are known to significantly modify the polarity of their environment. Even though our coarse grained (CG) simulations cannot predict the local dielectric constant, closely related to the local polarity, they report a non
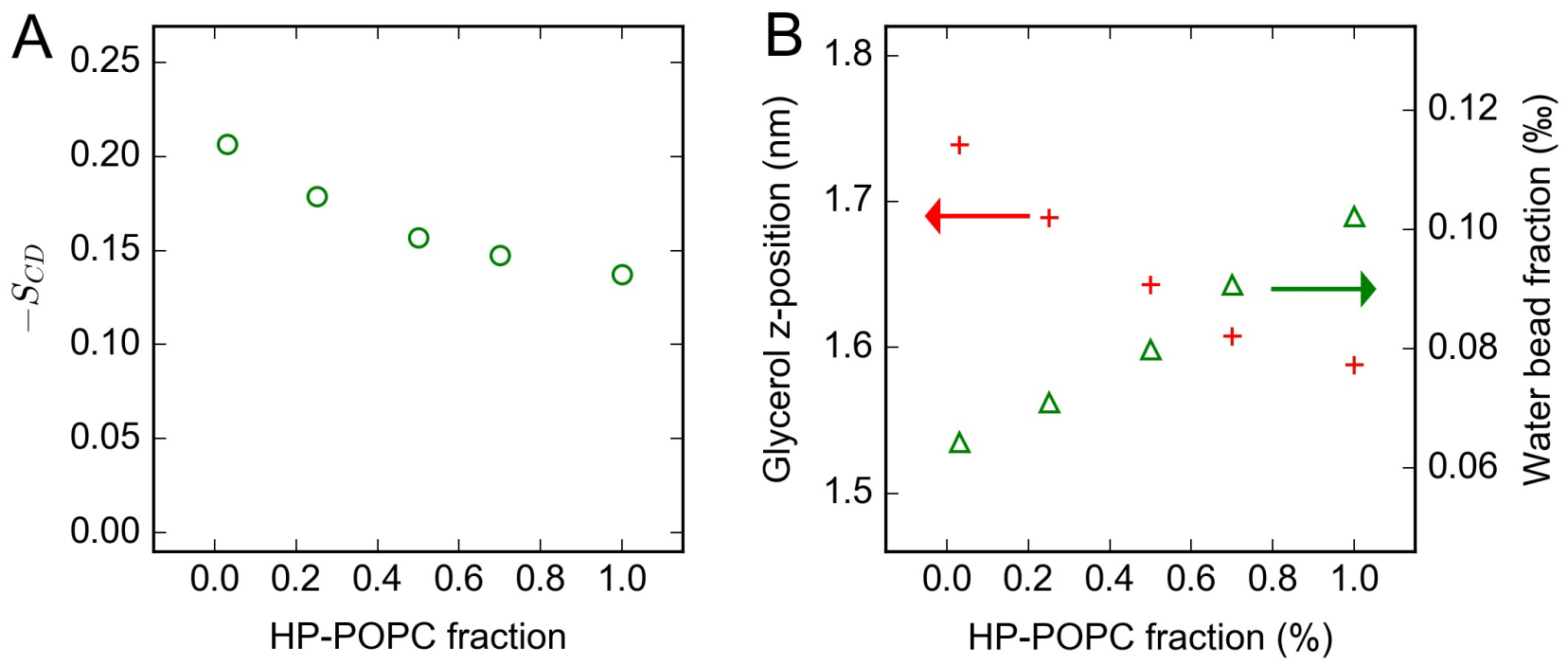

homogeneous distribution of the hydroperoxides along the z-direction in the hydrophobic region of the bilayer. Predictions from our model are summarized in Figures 9 and 10. Fig. 9 shows the $z$-dependent density curves for the - $\mathrm{OOH}$ group (thick lines). They suggest that at low oxidation ratio, hydroperoxyl groups are attracted to the interfacial region of the membrane, while for fully oxidized bilayers, they spread evenly between hydrophobic core (frontier being arbitrarily set to $z_{c}=+/-0.8$ $\mathrm{nm})$ and interfacial region $\left(z_{c}<z<z_{i}=2.5 \mathrm{~nm}\right)$ (Fig. 9). As a consequence, the local density of hydroperoxides in the hydrophobic core increases non-linearly with the oxidation rate as shown in Fi- 
gure 10 that reports the partitioning between core and interface. Note for example that at 50\% HPPOPC content, roughly only $30 \%$ of the $\mathrm{OOH}$ groups are located deep in the membrane (Fig. 10B).

Figure 8: A) Average order parameter $-S_{C D}$ (see 2.8), for various fractions of HP-POPC in the membrane. B) Average z-position of the glycerol bead (bead n³, see Fig. 4) and number of water beads per thousand lipids in the inner region of the bilayer, i.e. limited by the glycerol bead.

Figure 9: Number density of hydroperoxide groups for various oxidation ratio (thick lines), i.e. sta $^{-}$ tistics of positions of beads 12, see Figure 4. Density of hydroperoxides are normalized in such a way that their integration with respect to $z$ equals the percentage $\mathrm{N}$ of oxidized lipids $(\mathrm{N}=3,25,50$, 70 and 100). Dotted vertical lines are for $z=0$ (midplane), $z=z_{c}= \pm 0.8 \mathrm{~nm}$ (core limits), and $z=z_{i}= \pm 2.5$ $\mathrm{nm}$ (bilayer interfaces). Thin continuous lines: normalized density of water beads for the $3 \%$ and $100 \%$ peroxidized membrane (arrow oriented from 3\% towards 100\%).
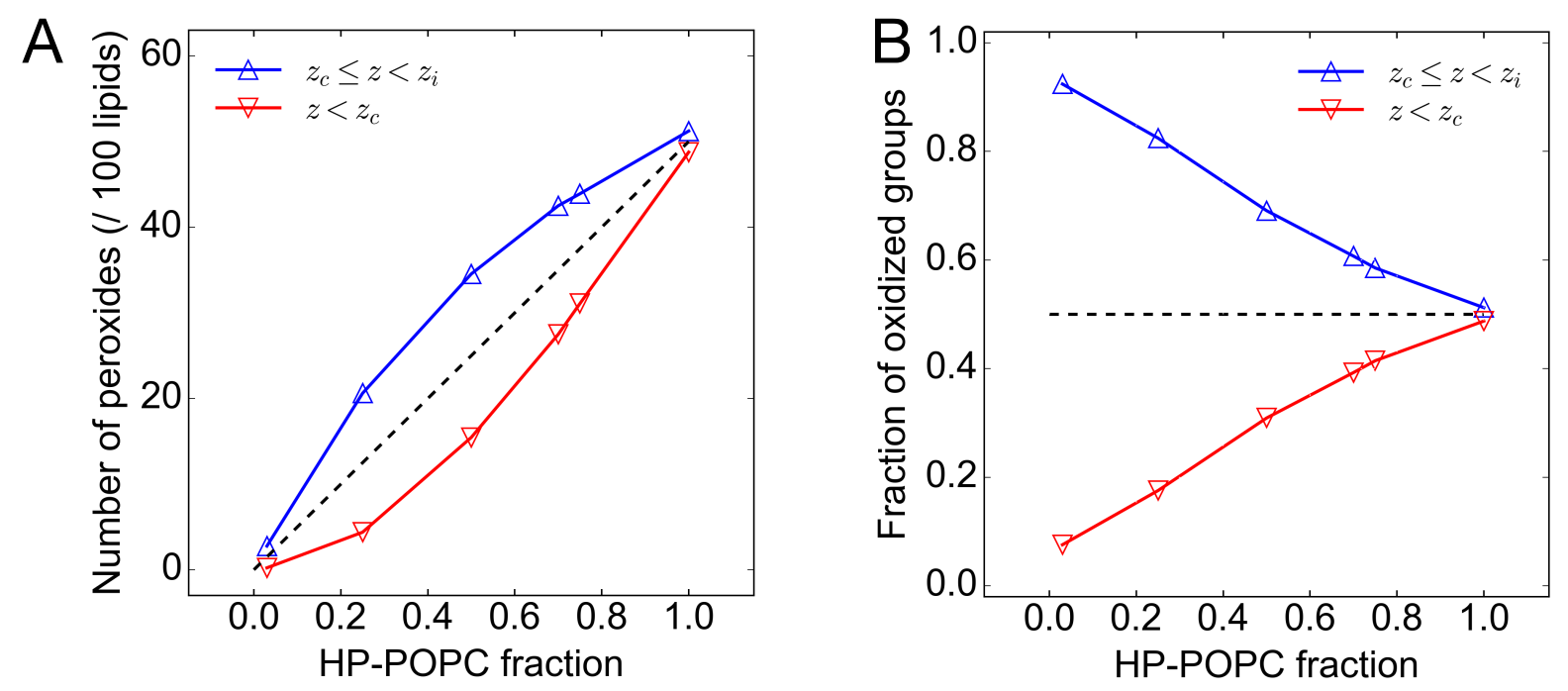

Figure 10: (A) number of hydroperoxide groups in core (red ' $\mathrm{v}$ ' triangles) and interfacial region (blue '^' triangles), for various fractions of HP-POPC. The sum of the two curves is equal to the percentage $\mathrm{N}$ of hydroperoxidation. (B) partition probabilities between core and interface as a function of the oxidized fraction. In both figures, the dotted line corresponds to an homogeneous distribution over the $z$-direction. At low concentration, most hydroperoxides stay at the interface while at large concentration they distribute evenly between core and interface. Note the affine increase of the core partitioning probability as a function of the hydroperoxidation fraction (B), consistent with a non-linear increase of the hydroperoxide density in the core region (A).

Thus, assuming that changes in the probe fluorescence properties are related to the close proximity of hydroperoxyl groups (either through a direct $\mathrm{OOH} / \mathrm{N}^{*}$ interaction, or through a lipid 
disorder-induced water/ $\mathrm{N}^{*}$ interaction), our numerical data (Fig. 8, 9, 10) are consistent with a nonlinear dependence of those properties with the oxidation ratio, something clearly highlighted by POL evolution with HP-POPC fraction (Fig. 6A).

Other studies based on numerical simulations have pointed out such non-linear evolution of the density distribution of the peroxide group along the $z$-direction. A good example is given in [41] for DLPC, a lipid with two doubly unsaturated carbon chains, where it is shown how the position of the $-\mathrm{OOH}$ group along the chain influences that distribution. In particular, for -OOH localized on the ninth position (EE9 in ${ }^{[41]}$ ), the peroxide group preferentially locates at the membrane-water interface whatever the peroxidation degree, corresponding thus to a stronger trend of expulsion of the -OOH towards the interface than what is observed for POPC (Fig. 9). On the contrary, for -OOH localized on the thirteenth position (EE13 in [41]), $-\mathrm{OOH}$ distribution shows a stronger localization probability in the core region than the one observed for POPC (Fig. 9). Thus, the behavior of POPC depicted in Fig. 9 appears as intermediate between the EE6 and EE13 cases studied in [41].

The discussion about location of the -OOH groups is of course of utmost importance, as for it has direct consequences for the membrane structural features. Rare studies have yet been performed that track the distribution of the peroxide group along the membrane $z$-direction. In particular, De Rosa et al. confirmed the migration to the membrane interface of -OOH groups, but with no evidence for inner membrane enrichment.[42] We also note that another coarse-grained model of HPPOPC was introduced by Siani et al.[43] This model, based on the ELBA lipid force-field[44] differs first in the way water molecules are represented, and secondly on the representation of the peroxide group. Indeed, hydroperoxidation of POPC in Siani et al. does not consist in adding an extra $-\mathrm{OOH}$ side chain, but in changing one of the oleoyl chain bead, analogue to bead number 10 of POPC in Figure 4. The resulting distribution of -OOH for a fully peroxidized chain is narrower than in Fig. 9, and unimodal (maximum probability located at $9 \mathrm{~nm}$ ).[43] However, Siani et al. do not show the density probability evolution with peroxidation degree, limiting the comparison between our two models.

The water distribution across the bilayer is represented in Fig. 8B and Fig. 9. The normalized water densities for 3\% and 100\% peroxidation are shown in Fig. 9, the intermediate values being not shown. One notes a significative increase of water density in the interface region, together with an increase of circa $0.1 \mathrm{~nm}$ of the penetration depth with peroxidation, see arrow on Fig. 9. Fig. 8B presents the evolution of the $z$-position of the glycerol bead with HP-POPC fraction and the 
concomitant evolution of the water/lipid fraction. Glycerol position decreases from $1.75 \mathrm{~nm}$ to circa $1.6 \mathrm{~nm}$, corresponding to a slight thinning of the bilayer with hydroperoxidation, in agreement with previous studies.[11] The number of water beads per thousand lipids in the inner part of the bilayer, i.e. as limited by the glycerol bead position, is roughly doubled for a $100 \%$ hydroperoxidized membrane (Fig. 8B). Water representation in Martini-type simulations should be considered with care. In particular, each bead in the present model represents four water molecules. Knowing that the energy barrier for a water molecule to cross a bilayer is in the order of a few tens of $k_{B} T,{ }^{[45]}$ the calculated numbers in Fig. 8B, particularly as far as the core region is concerned, should only be used as an indication of a qualitative trend, i.e. that though water presence in the core region increases with peroxidation, remaining however at a low level overall. This assertion is thus in agreement with the slight decrease of the order parameter $-S_{C D}$ (Fig. 8A). 


\section{Conclusions}

Hydroperoxidation of unsaturated lipids induces important changes in bilayer structure and properties.[10-12,14,15-17,19,37,41-43] Experimental evidence suggests that the average lipid area is not simply related to the fraction of hydroperoxidized lipids in the bilayer.[19,42] For instance, the hydroperoxidation of DOPC bilayers should be accompanied by a much larger increase in area than POPC bilayers, the unsaturation number being twice larger for the first, which is not seen experimentally.[19] As a matter of fact, SAXS analysis on liposomes show that the -OOH moiety preferentially locates towards the polar region of each monolayer of HP-POPC membranes, inducing an area per lipid increase well above the value expected from the geometrical rule that correlates an isotropic volume increase to the corresponding area increase.[42] The present work demonstrates that POPC bilayers are subject to significant structural reorganization as the ratio of hydroperoxidized molecules increases, and that the changes are not marginal neither just proportional to the average density of hydroperoxide groups. Martini numerical simulations show that in HP-POPC bilayers, the grafted organic hydroperoxide group -OOH not only migrates to the lipid bilayer-water interface but also populates deeper regions of the hydrophobic part of the bilayer for the higher -OOH fractions. Fluorescence emission deconvolution for the three fluorescent probes F2N12S, F66, and PPZ8, designed to incorporate at various depths in the membrane, all report a non linear increase of the local polarity, consistent with such concentration-dependant distribution of the -OOH groups along the $z$-direction of the bilayer. Interestingly, simulations and fluorescence are consistent i) with a relatively low modification of the lipid ordering, and ii) with low water penetration, whatever the peroxidation degree. POPC is abundant in many membranes of eukariotic species. As far as the biological realm is concerned, our results anticipate a strong influence of hydroperoxidation on the interaction of biomembranes with membrane proteins and with membrane-active biomolecules.[46] Indeed, while enrichment of the head region by -OOH groups will likely enhance the affinity of charged species with the membrane, an increased polarity of the inner membrane is expected to facilitate membrane crossing by species such as cell penetrating peptides. 


\section{Conflict of interest}

There are no conflicts to declare.

\section{Acknowledgments}

The authors thank Pr. Rosangela Itri for insightful discussions and for sharing non published material. Fundação de Amparo à Pesquisa do Estado de São Paulo (FAPESP) (2012/50680, 2013/07937-8, 2013/11640-0) and NAP-Phototech are acknowledged for financial support. Y. M. is grateful to the Institut Universitaire de France (IUF) for support and providing additional time to be dedicated to research. 


\section{References}

[1]

B.N. Ames, M. K. Shigenaga, T.M. Hagen, Oxidants, antioxidants, and the degenerative diseases of aging, Proc. Natl. Acad. Sci. U.S.A. 90 (1993), 7915-7922.

[2]

B. Halliwell, J.M.C. Gutteridge, Free radicals in biology and medicine, Oxford University Press, Oxford, 4th edn, 2007.

[3]

E.L. Crockett, The cold but not hard fats in ectotherms: consequences of lipid restructuring on susceptibility of biological membranes to peroxidation, a review, J. Comp. Physiol. B 178 (2008), 795809.

[4]

H. Sies, Biochemistry of oxidative stress, Angew. Chem., Int. Ed. Engl. 25 (1986), 1058-1071.

[5]

S.P. Stratton, D.C. Liebler, Determination of singlet oxygen-specific versus radical-mediated lipid peroxidation in photosensitized oxidation of lipid bilayers: effect of $\beta$-Carotene and $\alpha$-Tocopherol, Biochemistry 36 (1997), 12911-12920.

[6]

D.A. Pratt, J.H. Mills, N.A. Porter, Theoretical calculations of carbon-oxygen bond dissociation enthalpies of peroxyl radicals formed in the autoxidation of lipids, J. Am. Chem. Soc. 125 (2003), 5801-5810.

[7]

H.-P. Deigner, A. Hermetter, Oxidized phospholipids: emerging lipid mediators in pathophysiology, Curr. Opin. Lipidol. 19 (2008), 289-294.

[8]

R.S. Sohal, R. Weindruch, Oxidative stress, caloric restriction, and aging, Science 273 (1996), 59-63.

[9]

E. Niki, Y. Yoshida, Y. Saito, N. Noguchi, Lipid peroxidation: mechanisms, inhibition, and biological effects, Biochem. Biophys. Res. Commun. 338 (2005), 668-676. 
J. Wong-Ekkabut, Z. Xu, W. Triampo, I.-M. Tang, D. P. Tieleman, and L. Monticelli, Effect of lipid peroxidation on the properties of lipid bilayers: a molecular dynamics study, Biophysical Journal 93 (2007), 4225-4236.

[11]

Y. Guo, V.A. Baulin, and F. Thalmann, Peroxidised phospholipid bilayers: insight from coarsegrained molecular dynamics simulations, Soft Matter 12 (2016), 263-271.

W. Caetano, P.S. Haddad, R. Itri, D. Severino, V.C. Vieira, M.S. Baptista, A.P. Schroder, C.M. Marques, Photo-Induced Destruction of Giant Vesicles in Methylene Blue Solutions, Langmuir 23 (2007), 1307-1314.

O. Mertins, I.O.L. Bacellar, F. Thalmann, C.M Marques, M.S. Baptista, and R. Itri, Physical damage on giant vesicles membrane as a result of methylene blue photoirradiation, Biophysical Journal 106 (2014), 162-171.

[14]

K.A. Riske, T.P. Sudbrack, N.L. Archilha, A.F. Uchoa, A.P. Schroder, C.M. Marques, M.S. Baptista, R. Itri, Giant vesicles under oxidative stress induced by a membrane-anchored photosensitizer, Biophysical Journal 97 (2009), 1362-1370.

J. Heuvingh and S. Bonneau, Asymmetric oxidation of giant vesicles triggers curvature-associated shape transition and permeabilization, Biophysical Journal 97 (2009), 2904-2912.

H. Mojzisova, S. Bonneau, P. Maillard, K. Berg, D. Brault, Photosensitizing properties of chlorins in solution and in membrane-mimicking systems, Photochem. Photobiol. Sci. 8 (2009), 778-787.

[17]

R. Kerdous, J. Heuvingh, S. Bonneau, Photo-dynamic induction of oxidative stress within cholesterol-containing membranes: Shape transitions and permeabilization, Biochimica et Biophysica Acta 1808 (2011), 2965-2972. 
S. Sankhagowit, S.-H. Wu, R. Biswas, C.T. Riche, M. L. Povinelli, N. Malmstadt, The dynamics of giant unilamellar vesicle oxidation probed by morphological transitions, Biochimica et Biophysica Acta 1838 (2014), 2615-2624 .

[19]

G. Weber, T. Charitat, M.S. Baptista, A.F. Uchoa, C. Pavani, H.C. Junqueira, Y. Guo, V.A. Baulin, R. Itri, C.M. Marques, A.P. Schroder, Lipid oxidation induces structural changes in biomimetic membranes, Soft Matter (2014), 10, 4241-4247.

[20]

P.H.B. Aoki, A.P. Schroder, C.J.L. Constantino, C.M. Marques, Bioadhesive giant vesicles for monitoring hydroperoxidation in lipid membranes, Soft Matter 11 (2015), 5995-5998.

[21]

A.P. Demchenko, Y. Mély, G. Duportail, A.S. Klymchenko, Monitoring biophysical properties of lipid membranes by environment-sensitive fluorescent probes, Biophysical Journal 96 (2009), 3461-3470.

A.S. Klymchenko, Solvatochromic and fluorogenic dyes as environment-sensitive probes: design and biological applications, Acc. Chem. Res. 50 (2017), 366-375.

Y. Mély, G. Duportail (eds.), Fluorescent Methods to Study Biological Membranes, Springer Ser Fluoresc 13 (2013) 51-70, Springer-Verlag Berlin Heidelberg, 3-Hydroxychromone Probes Precisely Located and Oriented in Lipid Bilayers: A Toolkit for Biomembrane Research, A.S. Klymchenko, G. Duportail, Y. Mély.

A.S. Klymchenko, A.P. Demchenko, Multiparametric probing of intermolecular interactions with fluorescent dye exhibiting excited state intramolecular proton transfer, Phys. Chem. Chem. Phys. 5 (2003), 461-468.

[25]

A.P. Demchenko, K.-C. Tang, P.-T. Chou, Excited-state proton coupled charge transfer modulated by molecular structure and media polarization, Chem. Soc. Rev. 42 (2013), 1379-1408. 
A.S. Klymchenko, G. Duportail, T. Ozturk, V.G. Pivovarenko, Y. Mély, A.P. Demchenko, Novel two-band ratiometric fluorescence probes with different location and orientation in phospholipid membranes, Chem. Biol. 9 (2002), 1199-1208.

[27]

A.S. Klymchenko, Y. Mély, A.P. Demchenko, G. Duportail, Simultaneous probing of hydration and polarity of lipid bilayers with 3-hydroxyflavone fluorescent dyes, Biochim Biophys Acta. 1665 (2004), 6-19.

[28]

V.V. Shynkar, A.S. Klymchenko, C. Kunzelmann, G. Duportail, C.D. Muller, A.P. Demchenko, J.M. Freyssinet, Y. Mély, Fluorescent biomembrane probe for ratiometric detection of apoptosis, JACS 129 (2007), 2187-2193.

[29]

Z. Darwich, O.A. Kucherak, R. Kreder, L. Richert, R. Vauchelles, Y. Mély, A.S. Klymchenko, Rational design of fluorescent membrane probes for apoptosis based on 3-hydroxyflavone, Methods Appl. Fluoresc. 1 (2013), 025002.

[30]

V.V. Shynkar, A.S. Klymchenko, G. Duportail, A.P. Demchenko, Y. Mély, Two-color fluorescent probes for imaging the dipole potential of cell plasma membranes, Biochimica et Biophysica Acta 1712 (2005), 128-136.

M.I. Angelova, D.S. Dimitrov, Liposome electroformation, Faraday Discuss. Chem. Soc. 81 (1986), 303-311.

A.G. Ayuyan, F.S. Cohen, Lipid Peroxides Promote Large Rafts: Effects of Excitation of Probes in Fluorescence Microscopy and Electrochemical Reactions during Vesicle Formation, Biophysical Journal 91 (2006), 217.

J. Zhao, J. Wu, H. Shao, F. Kong, N. Jain, G. Huntb, G. Feigenson, Phase studies of model biomembranes: Macroscopic coexistence of $\mathrm{L} \alpha+\mathrm{L} \beta$, with light-induced coexistence of L $\alpha+\mathrm{Lo}$ Phases, Biochimica et Biophysica Acta 1768 (2007), 2777. 
D.B. Siano, D.E. Metzler, Band shapes of the electronic spectra of complex molecules, The Journal of Chemical Physics 51 (1969), 1856-1861.

A.S. Klymchenko, G. Duportail, A.P. Demchenko, Y. Mély, Bimodal distribution and fluorescence response of environment-sensitive probes in lipid bilayers, Biophysical Journal 86 (2004), 2929-2941.

S.J. Marrink, H.J. Risselada, S. Yefimov, D.P. Tieleman, A.H. de Vries, The MARTINI force field: coarse grained model for biomolecular simulations, The Journal of Physical Chemistry B 111 (2007), 7812-7824.

J. Van der Paal, E.C. Neyts, C.C.W. Verlackt, A. Bogaerts, Effect of lipid peroxidation on membrane permeability of cancer and normal cells subjected to oxidative stress, Chemical Science 7 (2016), 489.

[38]

A.S. Klymchenko, S. Oncul, P. Didier, E. Schaub, L. Bagatolli, G. Duportail, Y. Mély, Visualization of lipid domains in giant unilamellar vesicles using an environment-sensitive membrane probe based on 3-hydroxyflavone, Biochimica et Biophysica Acta (BBA): Biomembranes 1788 (2009), 495-499.

[39] : V.J. Greiner, C. Egelé, S. Oncul, F. Ronzon, C. Manin, A. Klymchenko, Y. Mély, Characterization of the lipid and protein organization in HBsAg viral particles by steady-state and time-resolved fluorescence spectroscopy, Biochimie 92 (2010), 994-1002.

[40]

V. Kilin, O. Glushonkov, L. Herdly, A. Klymchenko, L. Richert, Y. Mély, Fluorescence lifetime imaging of membrane lipid order with a ratiometric fluorescent probe, Biophys. J. 108 (2015), 2521-2531.

L. Rems, M. Viano, M.A. Kasimova, D. Miklavčič, M. Tarek, The contribution of lipid peroxidation to membrane permeability in electropermeabilization: A molecular dynamics study, Bioelectrochemistry 125 (2019), 46-57. 
R. De Rosa, F. Spinozzi, R. Itri, , Hydroperoxide and carboxyl groups preferential location in oxidized $\mathrm{T}$ biomembranes experimentally determined by small angle $\mathrm{X}$-ray scattering: Implications in membrane structure, BBA Biomembranes 1860 (2018), 2299-2307.

P. Siani, R. de Souza, L. Dias, R. Itri, H. Khandelia, An overview of molecular dynamics simulations of oxidized lipid systems, with a comparison of ELBA and MARTINI force fields for coarse grained lipid simulations, Biochimica et Biophysica Acta (BBA): Biomembranes 1858 (2016), 2498-2511.

[44]

M. Orsi, J.W. Essex, The ELBA force field for coarse-grain modeling of lipid membranes, PLOS ONE 6 (2011), 1-22.

S.J. Marrink, H.J.C. Berendsen, Permeation process of small molecules across lipid membranes studied by molecular dynamics simulations, J. Phys. Chem. 100 (1996), 16729-16738.

[46]

M.D.J. Libardo, T.Y. Wang, J.P. Pellois, A.M. Angeles-Boza, How does membrane oxidation affect cell delivery and cell killing?, Trends Biotechnol. 35 (2017), 686-690. 